Z. klin. Chem. u. klin. Biochem.

8. Jg., S. 507-515, September 1970

\title{
Zum Krankheitsbild der Lipodystrophia intestinalis (WHIPPLE) Fallbericht mit lipidchemischen Organbefunden
}

\author{
Von K. O. Schmid ${ }^{1}$ ), P. Spath, D. Eberhagen und M. Ciresa \\ Aus dem Pathologischen Institut (damaliger suppl. Leiter: Doz. Dr. K. O. Schmid) und der Medizinischen Klinile \\ (Direktor: Prof. Dr. H. Braunsteiner) der Universität Innsbruck und der Medizinischen Poliklinik
}

(Direktor: Prof. Dr. W. Seitz) der Universität München

(Eingegangen am 23. April 1970)

Bericht über einen seit 20 Jahren an Diarrhoen erkrankten 51 jährigen Mann. Erkrankung 10 Jahre klinisch als sprueartiges Malabsorptionssyndrom bekannt. Durch symptomatische Therapie und glutenfreie Diät etwa 6 Jahre Remission. Einige Monate vor dem Tode progressive Verschlechterung, Exitus an unstillbaren Magenschleimhauterosionsblutungen.

Obduktion und histologische Untersuchung ergeben Spätform des M. WhippLE. Chronische, auf weite Strecken atrophisierende Enterocolitis, auffallend geringer Schaumzellbefall des Dünndarms, spärliche PAS-positive Makrophagen, jedoch kein sicherer Bakteriennachweis.

Umwandlung der Mesenteriallymphknoten in bis nußgroße Pseudozysten mit Milch- bzw. quarkähnlichem Inhalt. Starke Stauung der mesenterialen Lymphgefäße, chylöser Aszites, chronische vernarbende Hepatitis.

Ergebnisse der lipid-chemischen Untersuchung: im Dünndarm starke Vermehrung der Gesamtlipide einschließlich Triglyceriden und freien Fettsäuren, deutliche Verminderung der Phosphatide. In der Leber deutliche Verminderung der Gesamtlipide, Gesamtcholesterin etwa unverändert, Triglyceride und Phosphatide vermindert. In den mesenterialen Lymphknoten Cholesterin sicher, Phosphatide wahrscheinlich erhöht. Einige Veränderungen im Fettsäuremuster einzelner Lipidfraktionen. Unter den Serumlipiden sind Cholesterin, Triglyceride und Phosphatide deutlich erniedrigt. Ein ungewöhnliches, konstitutionell unklares Lipid der Darmwand wurde vorläufig als Substanz X bezeichnet.

Obwohl durch die eigenen Untersuchungen keine Klärung der Atiologie und Pathogenese des M. WhippLE gelang, spricht viel für eine Resorptionsstörung des Dünndarmepithels, möglicherweise kombiniert mit einer (vermutlich nachgeschalteten) Störung des Chylomikronentransportes. Die Bedeutung des in der Literatur besonders hervorgehobenen Bakterienbefalles ist unklar.

\section{The pathology of Lipodystrophia intestinalis (WHIPPLE). Case report with lipid analyses of organs}

This is a case report of a 51 year old man hospitalized several times with a 20 year history of diarrhea and symptoms of sprue-like malabsorption starting 10 year ago. By symptomatic therapy and a gluten-free diet, the patient achieved remission for 6 years. Progessive deterioration developed several months prior to death which was caused by severe gastro-intestinal bleeding.

The autopsy revealed an advanced case of Whipple's disease (intestinal lipodystrophy). Histological examination showed a chronic, mainly atrophic enterocolitis, strikingly little mucosal infiltration with foam-cells, a few PAS + macrophages, but no evidence of bacteria.

There was transformation of the mesenteric lymph-nodes into pseudocysts varying in size up to a walnut, containing milky and curdlike material. Marked stasis of mesenteric lymph-nodes, chylous ascites and a chronic scarring hepatitis were found.

In the lipid-chemical analyses the small intestine showed a marked increase of the total lipids including triglycerids and free fatty acids and a distinct decrease of phospholipids. In the liver there was a marked decrease of total lipids, probably no abnormalities of cholesterol and a decrease of triglycerids and phospholipids. In the mesenteric lymph-nodes a definite increase of cholesterol was noted and a probable increase of phospholipids. There were abnormalities in the fatty-acid pattern of some of the lipid fractions. The serumlipids showed a distinct decrease of cholesterol, triglycerides and phospholipids. An abnormal lipid, chemically not yet completely defined, was found in the mucosa and provisionally called "substance X".

Although these investigations are not able to determine the etiology and pathogenesis of Whipple's disease, they suport the view that there exists an impaired absorption of fats, possibly combined with a block in the transport of chylomicrons. The significance of the bacterial infiltration, which is presumed to be of great etiological importance in some of the recent literature, is still unclear.

Obwohl die Erstbeschreibung durch WHIPPLE 1907 (2) schon mehr als 60 Jahre zurückliegt und die Zahl bioptischer Darmschleimhautuntersuchungen zugenommen hat, ist der Morbus W WIPPLE noch immer, trotz genauer Kenntnis seiner Klinik und pathologischen Anatomie, selten. Seine Ätiologie und Pathogenese ist umstritten. Im Vordergrund des pathologisch-anatomischen Geschehens stehen bekanntlich Schaumzellherde in der Dünndarmschleimhaut und mesenteriale Lymphknotenschwellung mit Verzystung, sowie Ansammlung

1) Auszugsweise vorgetragen anläßlich der 53. Tagung der Deutschen Gesellschaft für Pathologie in Mainz vom 27. Mai bis 31. Mai 1969 (1). von Lipiden und Eiweißstoffen in den gestauten Lymphbahnen und umgebauten Lymphknoten. Da die chemische Identifizierung dieser Organlipide beim M. WHIPPLE bisher nur in wenigen Fällen erfolgte, haben wir uns im eigenen Fall darum bemüht.

Fallbericht: Pat. W. O.

Vorgeschichte:

Als Kind Rachitis, mit 31 Jahren erstmals Neigung zu Durchfällen bei sonst gutem Befinden. 10 Jahre später Verschlechterung des Allgemeinbefindens, Müdigkeit, Appetitverlust, Husten, Auswurf, Gewichtsverlust $10 \mathrm{~kg}$. 1 Jaht später veranlaßten stenokardische Beschwerden, Atemnot, Durst und Anämie erstmalig die Aufnahme in ein Krankenhaus, wo folgende Diagnose gestellt wurde: Spruesyndrom (erbsbreiartige, voluminöse Gärungs- 
stühle) mit Malabsorption, Hypoprothrombinämie und Eisenmangelanämic bei Dolicho-Megacolon und stark erweitertem Dünndarm mit abnormem Relief. Neurofibromatosis von Recklinghausen. Durch intensive symptomatische Therapie und glutenfreie Diät durch 6 Jahre gute Remission. Mit 51 Jahren rapide Verschlechterung, starke Diarrhoen, Exsikkose, Anämie und erstmaliges Auftreten von Hautnekrosen, septischen Temperaturen, zunehmende Kachexie und Gelenkbeschwerden. Bei Aufenthalten in verschiedenen Krankenhäusern während der letzten 10 Jahre wurden Antibiotika, Herzglykoside, Vitamine, Blutkonserven, Anabolika, Leber- und Colitisdiät, zuletzt auch Corticoide verordnet. In stark reduziertem Allgemeinzustand wurde der Patient an die Med. Univ. Klinik Innsbruck verlegt (Aufenthalt vom 4. 11. bis 9.11. 1966, Krankenblatt Nr. 1741/66).

\section{Status:}

51 jähriger Mann, $63 \mathrm{~kg} / 184 \mathrm{~cm}$, Kachexie, Ödeme, allgemeine bräunliche Hautpigmentierung, v. Recklinghausen'sche Neurifobromatose rechts mit „,café au lait“-Flecken, verstärkte Kyphose der BIWS, ulceröse Hautdefekte an Händen, Unterarmen, Knien und Unterschenkeln, Zungenulcera, vergrößerte Lymphknoten (Submandibulär, supraklavikulär, axillär und inguinal), Meteorismus (keine Milz- und Lebervergrößerung). Sacrales Dekubitalgeschwür.

EKG: Sinusrhythmus, supraventrikuläre Extrasystolie, linkspräkordiale Innenschichtalteration bei linksventrikulärer Leitungsrexzögerung.

Thoraxröntgerì: Mäßiges Emphysem, etwas nach rechts verbreitertes Herz, geringfügige Aortensklerose.

Laborbefunde: BSG 8/19, Luesreaktionen neg., Serum-Bilirubin direkt + , gesamt $0,8 \mathrm{mg} / 100 \mathrm{ml}$, Thymoltrübung $51 / 2 \mathrm{E}$., alkal. Phosphatase 20,0 i. E., 17 Uhr-Blutzucker $100 \mathrm{mg} / 100 \mathrm{ml}$, Harnstoff-N $17,5 \mathrm{mg} / 100 \mathrm{ml}$, Kalium 2,95 mKal $/ l$, Calcium 3,32 mKal $/ l$, Phosphor $1,0 \mathrm{mg}$-Atom $/ l$, Eisen $55 \mu \mathrm{g} / 100 \mathrm{~m} l$, latentes Eisenbindungsvermögen $165 \mu \mathrm{g} / 100 \mathrm{~m} l$, totales EBV $220 \mu \mathrm{g} / 100 \mathrm{~m} l$.

Harn: Eiwreiß stark opal, Glucose 0,25\%, Urobilinogen vermehrt, Sediment: mehrere Erythrocyten, einzelne Hefezellen, einige fettig degenerierte Rundepithelien, einige Plattenepithelien.

Blutbild: Erythrocyten 4,36 Mill/mm $\mathrm{mm}^{3}, \mathrm{Hb} 12,3 \mathrm{~g} / 100 \mathrm{ml}, \mathrm{HbE}$ $28,5 \mathrm{pg}$, Leukocyten $22000 \mathrm{~mm}^{3}$ Neutrophilie und Linksverschiebung, Neutrophile z. T. toxisch granuliert, 16,5\% Lymphocyten.

Elektropborese: Gesamteiweiß 4,3 g/100 ml, Albumine 34,7\%, $\alpha_{1}$-Glob. 5,7\%, $\alpha_{2}$-Glob. 10,2\%, $\beta$-Glob. 15,3\%, $\gamma$-Glob. 34,1\%. Serumlipide: Cholesterin $92 \mathrm{mg} / 100 \mathrm{ml}$, Triglyceride $85 \mathrm{mg} / 100 \mathrm{ml}$, freie Fettsäuren $680 \mu \mathrm{Val} / l$, Phosphatide $134 \mathrm{mg} / 100 \mathrm{ml}$.

Gleichzeitig mit septischen Temperaturen abgenommene Blutkulturen ergaben $2 \mathrm{mal}$ kein Bakterienwachstum, eine Harnkultur blieb ebenfalls steril.

Therapie

Die Bebandlung mit Cedilanid, Infusionen, Plasmakonserve, Vitaminen, Anabolikum, Elektrolytsubstitution und Butazolidin konnte den Zustand nicht bessern, Diarrhoen und septische Temperaturen bestanden weiterhin, der Patient verstarb an einer massiven gastrointestinalen Blutung.

Epikrise

Zusammenfassend hatte es sich also um ein mindestens 10 Jahre dauerndes, schließlich zur Kachexie führendes Malabsorptionssyndrom (sprueartig) gehandelt, welches durch Vitaminmangelerscheinungen, Anämie, Leberschädigung, hypoproteinämische Odeme, zeitweise rheumatoide Gelenkbeschwerden und ein nekrotisierendes Exanthem kompliziert war, schließlich zu einer tödlichen gastro-intestinalen Blutung führte.

Obduktionsbefund

(Auszug, L. Ö. Nr. 46189/1002/66 Obduzent Dr. HöLzL):

Als wesentlichster Befund eine Lipodystrophia intestinalis (Whipple'sche Exkrankung). In der freien Bauchhöhle etwa $500 \mathrm{ml}$ chylöser Aszites. Die Faltenzeichnung des Jejunums nur angedeutet vergröbert, keine gelben Stippchen der Schleimhaut. Ileumscbleimbaut hochgradig atrophisch, Payer'sche Plaques deutlich reduziert. Sigma und Rectum ödematös; Magenscbleimbaut mit multiplen, durchschnittlich linsengroßen, hämorrhagischen Erosionen; in der Lichtung des Magens und Dünndarms teichlich Blut. Die Lympblenoten des Dünndarmgekröses kettenförmig in bis pflaumengroße, prall elastische, kugelförmige Auftreibungen umgewandelt, die sich an Durchschnitten als glattwandige Zystoide erweisen, welche von milchigem Saft bzw. grauweißlich eingedickten, quarkartigen Massen exfüllt sind. Die abgehenden Lymphgefäße streckenweise bis über stricknadeldick, dadurch leicht zu eröffnen. Der Ductus thoracicus wegsam.

Leber etwa um ein Viertel verkleinert, oberflächlich und am Schnitt feinnarbig gefeldert, rötlich bis graugelblich gefleckt, Läppchenstruktur unscharf.

Milz nur hühnereigroß, Pulpa frisch aufgelockert. In der Rinde beider Nieren bis linsengroße, anämische, manchmal rötlich gesäumte Keile. Spongiosabalken der Wirbelkörper deutlich rarefiziert.

Haut: Ausgedehnte Neurofibromatose mit milchkaffeeartigen Flecken; vorwiegend an den Extremitäten bis linsengroße, oft borkenbedeckte unscharf begrenzte Nekrosen.

Zunge: Mehrere Nekrosen am Rand.

Herz: In Vorder- und Hinterwand des linken Ventrikels zahlreiche kleine Schwielen, das darüberliegende Epikard mit dem Perikard verwachsen.

\section{Histologische Untersuchung}

Im Jejunum (Abb. 1) dichte chronisch-entzündliche Infiltrate mit reichlich Plasmazellen und Lymphocyten, wechselnd reichlichen Makrophagen, darunter einige Schaumzellen und PAS-positive Elemente. Man vermißt hingegen dichte Schaumzellinfiltrate. Die zellige Durchsetzung am stärksten im Schleimhautstroma und in der Submukosa, hier deutliches Ödem. Die Chylusgefäße etwas dilatiert. Gegen das Ileum zunehmende Schleimhautatrophie, vielerorts dickdarmähnlicher Umbau mit reichlich Becherzellen. Im Dickdarm schwere, chronische, atrophisierende Entzündung. Starke Ausweitung der intramuralen Lymphwege, oft mit dichten perivaskulären Kleinrundzellinfiltraten, die auf das Gekröse übergreifen. Kleine Schaumzellherde submukös und subserös um die entzündlich gesäumten Lymphgefäße. Bakterienfärbungen im Dünndarm negativ, kein sicherer Anhaltspunkt für SPC-Zellen. Mesenteriale Lympllknoten: Veränderungen aller Stadien, doch Frühveränderungen seltener als Spätveränderungen. Im Rahmen der Lymphstauung schwammartiger Umbau (Abb. 2) gefolgt von Nekrose, Sequestrierung und zystischem Zerfall (Abb. 3a und $b$ ). In den Randbezirken wechselnd reichlich Sudan und PAS-positive Makrophagen. Der milchige und quarkartige
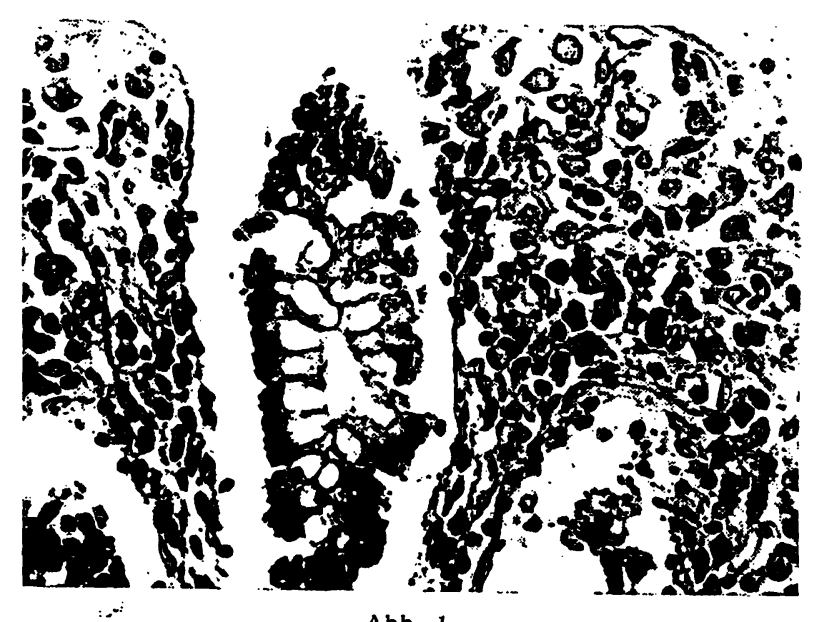

Abb. 1

Schaumzeliherde und starke chronische Entzündung im Jejunum Paraffinschnitt Haematoxylin-Eosin. Vergrößerung 270 fach 


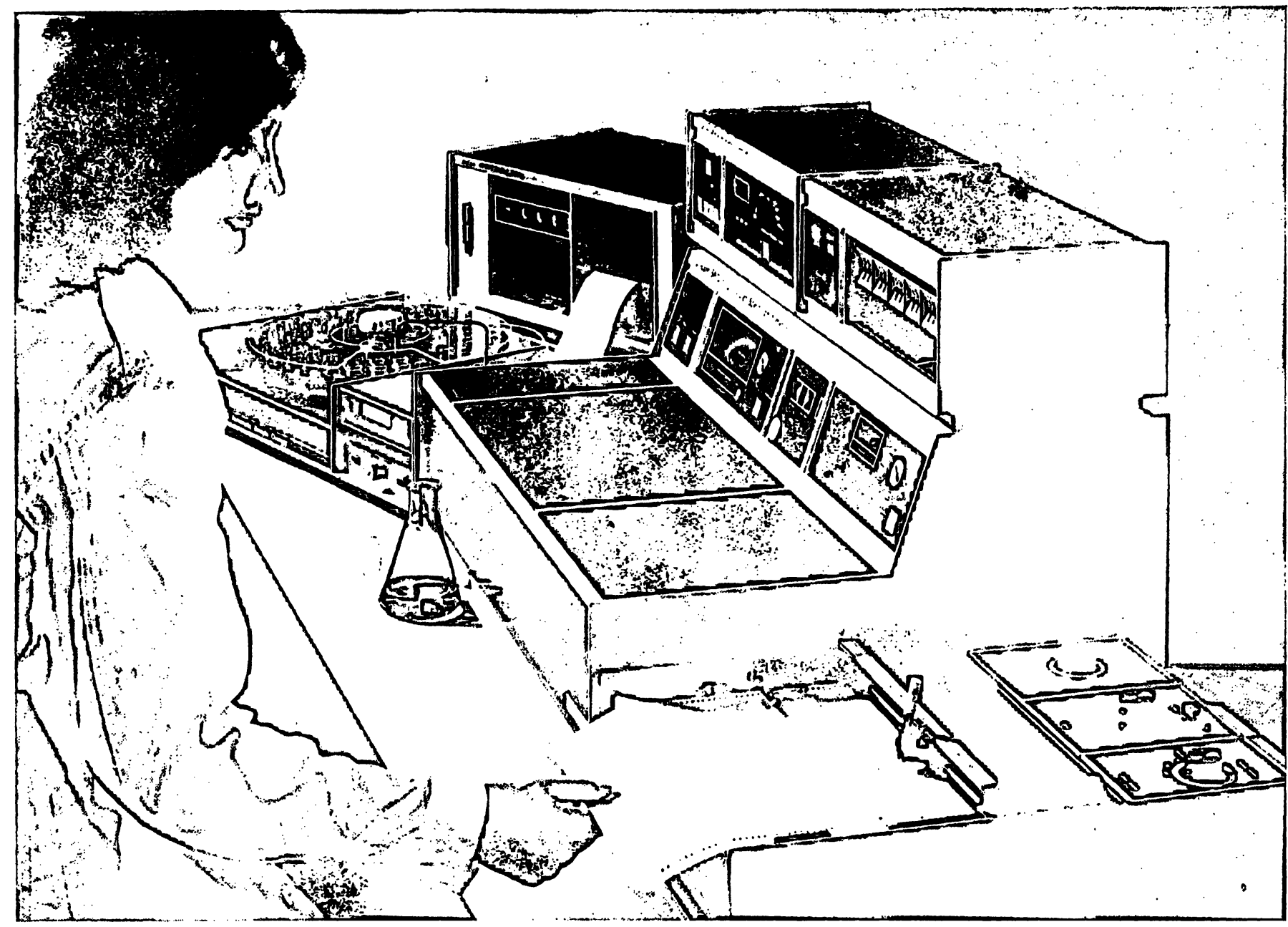

Sie analysieren vielseitiger, schneller, bequemer und erzielen genauere Ergebnisse mit dem UV-Spektralphotometer SP 1800 - eine Neuentwicklung aus dem Programm PYE UNICAM

\section{damit ist lhr Analysenproblem gelöst}

Das Doppelstrahl-Spektralphotometer für den ultravioletten und sichtbaren Bereich hat die hohe photometrische Genauigkeit von $\pm 0,002 \mathrm{E}$. Es wird als Grundgerät eingesetzt mit direkter Extinktionsanzeige auf eingebautem Zeigerinstrument mit 4 Extinktionsskalen $(0-0,2 \mathrm{E} ; 0-0,5 \mathrm{E} ; 0-1,0 \mathrm{E}$ und $0-2,0 \mathrm{E})$; umschaltbar für Konzentrationsanzeige; hochauflösendem Gitter-Monochromator; großem Probenraum und zweiter Probenstelle; automatischem Lampenwechsel.

Als vollautomatisches Spektralphoto-
meter-System bietet es Ihnen durch mehrere Zusatz-Einheiten weitere Vorteile:

Spektrendarstellung - komprimierte und gedehnte Spektren - durch synchronisierte Registrierung auf dem Schreiber AR 25; digitale Datenausgabe; wiederholte Registrierung eines Wellenlängenbereiches sowie bei mehreren automatisch vorwählbaren Wellenlängen; Mehrkanalbetrieb für kinetische Untersuchungen; automatischer Probenwechsler; besondere Schaltungen erlauben das Programmieren von wiederholten, ver- zögerten oder ferngesteuerten Messungen mehrerer Proben.

Ausführliches Informationsmaterial liegt für Sie bereit. Bitte fordern Sie es an.

Philips Elektronik Industrie GmbH 2000 Hamburg $63 \cdot$ Röntgenstraße 22 Telefon (0411) 501031

Büros In: Berlin Tel. (0311) 245908, Düsseldorf Tel. (0211) 346051, Dortmund Tel. (0231) 4 1961, Frankfurt Tel. (0611) 79131. Hannover Tol. (0511) 16601, München Tel. (0811) 76791 . Stuttgart-Follbach Tel. (0711) 589081 , Bielefeld Tel.
(0521) 23081 , Bremen Tol. (0421) 3100 41, Nürnberg Tel. (0911) 464763 .

PHILIPS

Analysengeräte UV-Spoktralphotometer SP 1800 und bitten um

Zusendung ausführlicher Unterlagen oln Angebot

olne unverbindliche Vorführung

Gewünschtes bitte ankreuzon oder crgänzen 
Herausgegeben vọn

Prof. Dr. med. Heinrich Bartelheimer

Direktor des I. Medizinischen Universituke-Klinik Hamburg

Prof. Dr. med. Hans-Joachim Maurer

Institut und Klinik flur Medizinische Stzahleakunde der Universitst Dusseldorf

Prof. Dr. med. Hans W. Schreiber

Chefarzt der Chirurgischen Abteilung

des Marien-Krankenhauses, Hamburg

Groß-Oktav. XVI, 513 Seiten.

Mit 210 Abbildungen und

2 mehrfarbigen Tafeln.

1969. Ganzleinen DM 88,- unter Mitwirkung von

Priv.-Doz. Dr. med. Kurt Müller-Wieland

Oberasze der I. Medizinischen Universitătr-Klinik Hamburg

\section{Inhaltsübersicht}

Die Probleme des Kranken mit operiertem Magen ( $\mathrm{H}$. Bartelheinar-Hamburg - H.-J. Maurer-Düsseldorf - H. W. SchreIBER-Hamburg)

\section{Magenresektion}

Indikationen zur Magenoperation und Darstellung der konventionellen und modernen Verfahren (L. ZuRSCHIVRDT und E. FARTHMANN-Hamburg) - Postoperative Frühkomplikationen nach einer Magenoperation (F. STELZNER-Hamburg)

\section{Der operierte Magen}

Physiologie und Pathologic des operierten Magens ( $\mathrm{H}$. W. Schreiber und K. Krentz-Hamburg) - Pathologische Anatomie des resezierten Magens (V. BeCKERBerlin) - Immunpathologie gastritischer Veränderungen (K. O. VORLAENDER-BONn)

Klinik des Magenresezierten

Der funktionell gut operierte Magen (K. MüLLER-WIELAND-Hamburg) - Subjektive und objektive Symptome (K. Müller-WIrLand-Hamburg) - Psychologische Aspekte der Magenoperation und des Magenoperierten (H. FrEYBERGER-Hamburg)

Diagnostik

Sekretionsanalyse (K. KRENTZ-Hamburg) - Gastrobiopsie (K. KRENTZ-Hamburg) - Zytologie (K. KRENTZ-Hamburg) - Gastroskopie (K. KRENTZHamburg) - Röntgenuntersuchung des Magenoperier- ten (H.-J. Maurer-Düsseldorf) - Nuklearmedizinische Untersuchungsmethoden ( $H$. BerNDT-BerlinBuch)

Spätsyndrom des Magenresezierten

Ernährungsstörungen, Magenstumpfkarzinom, Lungentuberkulose der Magenoperierten (K. MÜLLERWretaND-Hamburg) - Anämien nach Magenresektion und Gastrektomie (W. Pribilla-Berlin-Moabit) Skelett- und Calcium-Stoffwechselveränderungen nach Magenresektion (F. KUHLENCORDT-Hamburg) - Beeinflussung der Nachbarorgane (Leber, Galle, Pankreas) (E. WILDHIRT-Kassel)

Therapie bei Kranken mit Magenresektion

Therapie bei Kranken mit einer Magenoperation vom Standpunkt des Chirurgen (A. GürgemanN und H.W. Schrerber-Bonn-Hamburg) - Therapie bei Kranken mit einer Magenoperation vom Standpunkt des Internisten (M. GüLzow und K. Drwok-Rostock) - Operationsfehler (A. GütgemanN und H. W. SChreIBerBonn-Hamburg)

Besondere Gesichtspunkte bei magenoperierten Kindern (F. MrIssner-Leipzig)

Nachsorge und Begutachtung

Rehabilitation und Resozialisierung (A. LuCHMANNBad Salzig) - Begutachtung (Tr. O. LINDENSCHMrdTHamburg) 


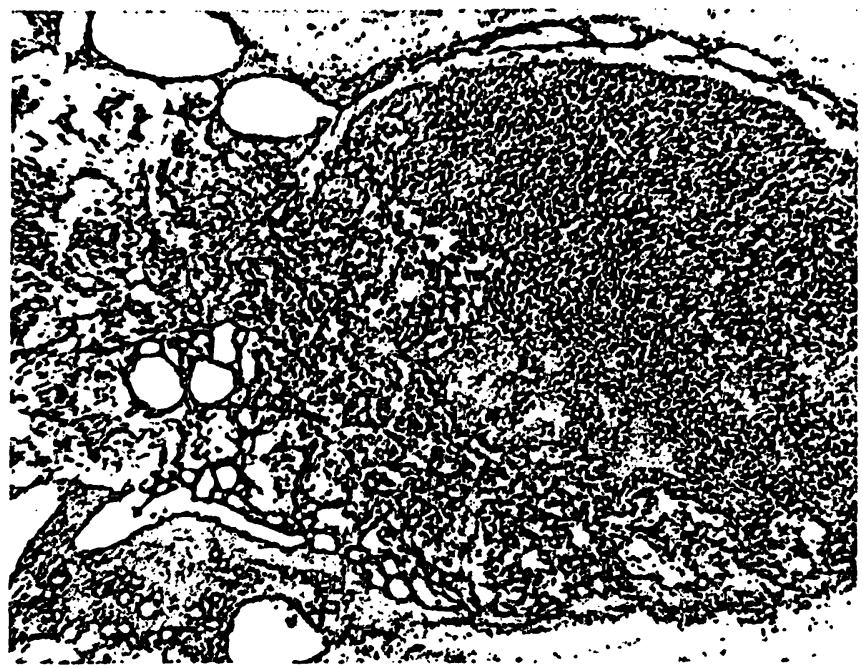

Abb. 2

Mesenteriallympliknoten, kleinzystischer Umbau infolge Lymphstauung (beginnendes "Schwammstadium") Paraffinschnitt.! PASFärbüg. Vergrößerung $48 \mathrm{fach}$

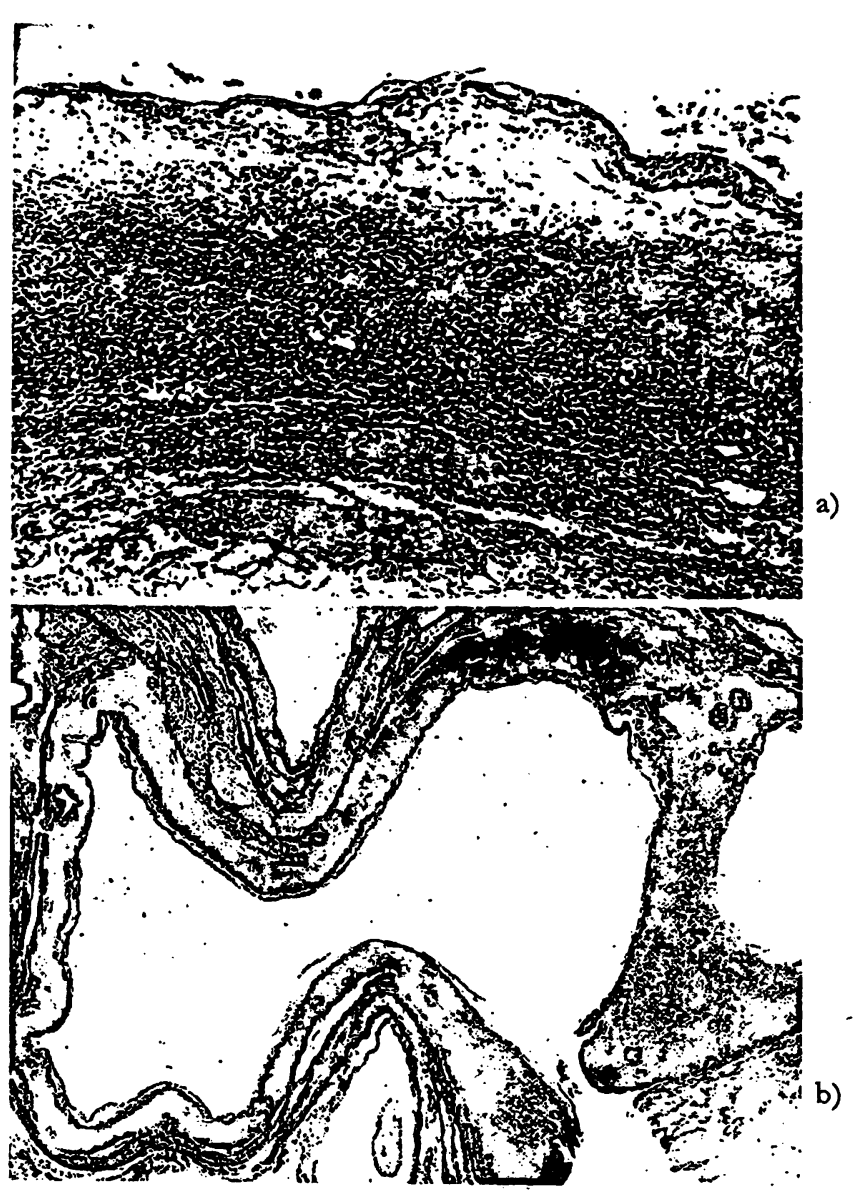

Abb. 3 a) Innere Zerfallszone eines mesenterialen Lymphknotenzystoids mit
auffallend wenigen Schaumzellen. Paraffinschnitt HaematoxylinEosin. Vergrößeriung 75 fach

b) Hochgradig verzysteter mesenterialer Lymphknoten mit schmalem Saum erhaltenen lymphoretikulären Restparenchyms. Paraffinschnitt Haematoxylin-Eosin. Vergrößerung 5,6 fach

Zystoidinhalt neutralfett-positiv gelegentlich schollig verkalkt. Durch den Druck der Inhaltsmasse Schwund des begrenzenden, lymphoretikulären Restgewebes (Abb. 3b). Schließlich restiert ein Zystoid mit derb fibrös-hyaliner Wand. Im Magen multiple hämorrhagische Erosionen bei schwerer chronischer Schleimhautentzündung. In der Leber chronische Entzündung mit Narbenbildung.
Ferner bestanden cine konfluicrte Bronchopneumonic im linken Unterlappen, vereiterte anämische Nicreninfarkte, cine ulcerösnekrotisicrende Glossitis sowic ausgedehntc Hautnckrosen (auf letztere wird in cincr weiteren Mittcilung speziell cingegangen).

Der Tod crfolgt an Verblutung aus multiplen hämorrhagischen Magenschlcimhautcrosionen.

\section{Lipidehemische Aufarbeitung}

Das Organmaterial wurde nach Zusat\% von $3,5 \mathrm{~m} /$ (Leher) bzw. 4,5 m/ (Darm) 0,9proz. NaCl-Lösung mit einem Messerhomogenisator zerkleinere und der entstandene Gewebsbrei in das 20 fache Volumen Chloroform-Mcthanol 2:1 (v/v) cingetragen.

Nach dreitägigem Stchen bei Rauntemperatur wurdc abfiltriert und das Filtrat durch Verteilung gegen Wasser nach Folch und Mitarbeitern (3) von den nicht lipoiden Extraktstoffen befreit. Die wäßr. obere und dic chlorsformhaltige untere Phase wurden im Rotationsverdampfer bei Badtemperaturen nicht über $40^{\circ}$ eingedainpft und die Rückstände im Vakuumexsikkator getrocknet. Der Destillationsrückstand der oberen Phase wird im folgenden als wasserlösliche Extraktstofie, der der unteren als Gesamtlipoide bezeichnet. Das Trocknen des extrahicrten Gewebsrückstandes erfolgte im Trockenschrank bei etwa $120^{\circ}$ bis zur Gewichtskonstanz. Dic erziclten Ausbcuten sind in der Tabelle 1 zusammengestellt.

Die aus der Leber (Tab. 2. u. 3) gewonnenen Gesamtlipoide wurden als 2,5proz. Lösung in Chloroform aufgenommen und

Tab. 1

Ausbeuten bei der lipidchemischen Aufarbeitung einer Gewebsprobe von Leber und Darm

\begin{tabular}{|c|c|c|c|}
\hline & & Leber & Darm \\
\hline \multirow{2}{*}{$\begin{array}{l}\text { Feuchtgewicht } \\
\text { Wasserlösliche Extraktstoffe } \\
\text { Trockengewiclit des extrahier- } \\
\text { ten Gewebsrückstands } \\
\text { Gesamtlipide } \\
\text { Trockengewicht } \\
\text { Gesamtlipide }{ }^{\circ} \text {; des Organtrocke }\end{array}$} & $\begin{array}{l}\text { (g) } \\
\text { (g) }\end{array}$ & $\begin{array}{l}6,601 \\
0,114\end{array}$ & $\begin{array}{l}8,164 \\
0,099\end{array}$ \\
\hline & $\begin{array}{l}\text { (g) } \\
\text { (g) } \\
\text { engewichtes) }\end{array}$ & $\begin{array}{l}0,970 \\
0,117 \\
1,201 \\
9,75\end{array}$ & $\begin{array}{r}0,388 \\
2,180 \\
2,667 \\
82\end{array}$ \\
\hline
\end{tabular}

Tab. 2

Zusammensetzung der Organlipide

\begin{tabular}{|c|c|c|c|c|}
\hline & \multicolumn{2}{|c|}{ Leber } & \multicolumn{2}{|c|}{ Darm } \\
\hline & $\begin{array}{l}\text { onder } \\
\text { Gesamt- } \\
\text { lipide }\end{array}$ & $\begin{array}{l}\text { ódes } \\
\text { Organ- } \\
\text { trocken- } \\
\text { gewichts }\end{array}$ & $\begin{array}{l}\text { Ooder } \\
\text { Gesamt- } \\
\text { lipide }\end{array}$ & $\begin{array}{l}\text { o' des } \\
\text { Organ- } \\
\text { trocken- } \\
\text { gewichts }\end{array}$ \\
\hline $\begin{array}{l}\text { Neutralfette } \\
\text { Cholesterinester } \\
\text { Freies Cholesterin } \\
\text { Triglyceride } \\
\text { Substanz X }\end{array}$ & $\begin{array}{l}5,10 \\
9,60 \\
4,60\end{array}$ & $\begin{array}{l}0,50 \\
0,94 \\
0,45 \\
\end{array}$ & $\begin{array}{r}0,91 \\
0,61 \\
88,8 \\
1,20\end{array}$ & $\begin{array}{r}0,75 \\
0,49 \\
72,55 \\
1,01\end{array}$ \\
\hline $\begin{array}{l}\text { Diglyceride } \\
\text { Monoglyceride } \\
\text { Freie Fettsäuren }\end{array}$ & $\begin{array}{l}0,25 \\
1,00 \\
6,25\end{array}$ & $\begin{array}{l}0,02 \\
0,10 \\
0,61\end{array}$ & \} $\begin{array}{l}0,91 \\
4,30\end{array}$ & $\begin{array}{l}0,75 \\
3,52\end{array}$ \\
\hline $\begin{array}{l}\text { Phosphatide } \\
\text { Cardiolipin, Phos- } \\
\text { phatidsäure u. ä. } \\
\text { Colaminkephalin } \\
\text { Lecithin } \\
\text { Sphingomyelin }\end{array}$ & $\begin{array}{r}+ \\
31,6 \\
38,5 \\
3,4\end{array}$ & $\begin{array}{l}+ \\
3,08 \\
3,75 \\
0,33\end{array}$ & $\begin{array}{l}+ \\
0,43 \\
1,08 \\
0,16\end{array}$ & $\begin{array}{l}+ \\
0,35 \\
0,88 \\
0,13\end{array}$ \\
\hline
\end{tabular}

Tab. 3

Zusammensetzung der aus den Leberlipidfraktionen gewonnenen Fettsäuregemische

\begin{tabular}{lrrrrr}
\hline $\begin{array}{c}\text { Fettsäure*) } \\
\text { [\%] }\end{array}$ & $\begin{array}{c}\text { Trigly- } \\
\text { ceride }\end{array}$ & $\begin{array}{c}\text { Freie } \\
\text { Fett- } \\
\text { säuren }\end{array}$ & $\begin{array}{c}\text { Phospha- } \\
\text { tidsäure } \\
\text { u. ä. }\end{array}$ & $\begin{array}{l}\text { Colamin- } \\
\text { kephalin }\end{array}$ & Lecithin \\
\hline $12: 0$ & 1,5 & 1,0 & 4,5 & & \\
$14: 0$ & 9,5 & 26,0 & 9,0 & 3,0 & 3,0 \\
$16: 0$ & 20,0 & 19,0 & 20,5 & 32,5 & 30,0 \\
$16: 1$ & 5,0 & 2,5 & 3,0 & 0,5 & 3,5 \\
$18: 0$ & 6,0 & 10,5 & 24,0 & 31,5 & 11,0 \\
$18: 1$ & 36,5 & 19,5 & 26,7 & 18,5 & 19,5 \\
$18: 2$ & 10,5 & 10,0 & 12,0 & 7,5 & 15,0 \\
$20: 0$ & 0,5 & 1,5 & & & 1,2 \\
$20: 3$ & 1,0 & 3,0 & & 2,0 & 9,5 \\
$20: 4$ & 2,5 & 3,5 & & 0,5 & 6,0 \\
$22: 0-6$ & 3,5 & 4,5 & & & \\
\hline
\end{tabular}

*) Die Zahl vor dem Doppelpunkt gibt die Kettenlänge, die Zahl nach dem Doppelpunkt die Zahl der vorhandenen Doppelbindungen an. 
aliquote Mengen zur Bestimmung des Phosphorgehaltes nach BARTLETT (4), des Gehaltes an freiem und Gesamtcholesterin nach $\mathrm{ZAK}_{\mathrm{AK}}$ und Mitarbeitern (5) und der Acylester nach FRIED und Hoeflatar 1963 (6) verwendet. Dic präparative Auftrennung geschah säulenchromatographisch mit Hilfe der von EBERHAGEN und Mitarbeitern (7) angegebcnen Technik. Die jeweils eingesetzte Substanzmenge betrug $25 \mathrm{mg}$. Als Adsorbens diente Kieselgel Merck mit einer Korngröße zwischen 0,05 und 0,2 mm; eluiert wurde mit der Lösungsmittelfolge B. Der Trennverlauf wurde dünnschichtchromatographisch nach ZöLLNER und WoLFRAM (8) (Neutralfette) bzw. WAGNER (9) oder SkIPSKr und Mitarbeiter (10) (Phosphatide) verfolgt. Die Leberlipide wurden auf diese Weise in sechs, allerdings meistens nicht einheitliche Fraktionen, zerlegt.

In der zuerst eluierten Fraktion ließen sich dünnschichtchromatographisch drei Substanzen mit ähnlichen $R_{\mathrm{F}}$-Werten wie Cholesterinester nachweisen. Die Salkowski-Reaktion (Rotfärbung nach Besprühen der Platten mit 50ptoz. wäßr. Schwefelsäure und anschließendem Erhitzen) gab jedoch nur die mittlere Bande. Um einen Anhalt über die quantitative Zusammensetzung zu erhalten, wurden die substanzhaltigen Kieselgelbezirke nach Sichtbarmachung der Banden mit Joddampf in Zentrifugengläser übertragen, $2 \mathrm{~m} /$ Chloroform zugegeben und die Lipoide, wie an anderer Stelle (EBERHAGEN und REMLER (11)) beschrieben, extrahiert. Ihre jeweiligen Mengen ließen sich mit Hilfe der Acylesterbestimmung ermitteln. Aufgrund einer Dreifachbestimmung ergab sich folgende Zusammensetzung: Dünnschichtchromatographisch fàsti mit der Lösungsmittelfront wandernde Bande A $10 \%$, Salkowski-positive Bande B $17 \%$ und Bande C $72 \%$.

Die zweite Säulenfraktion enthielt die Triglyceride. Sie war chromatographisch einheitlich. Ihr Substanzgehalt wurde nach Bestimmung der Acylesterbindungen errechnet, indem ein der Ölsäure entsprechendes mittleres Molekulargewicht der gebundenen Fettsäuren angenommen wurde. Für die gaschromatographische Untersuchung der Fettsäurenzusammensetzung (methodische Einzelheiten s. Eberhagen und Pletre (12)) wurde die Substanz mit methanolischer $\mathrm{HCl}$ umgeestert und die Methylester ror der Analyse säulenchromatographisch gereinigt (EBERHAGEN (13)).

Die dritte Säulenfraktion setzte sich aus mehreren Komponenten zusammen. Dünnschichtchromatographisch zeigten sich zwei ausgeprägte Banden mit $R_{\mathrm{F}}$-Werten, die den freien Fettsäuren und dem Cholesterin entsprachen - die Identität der letzteren konnte durch eine positive Salkowski-Reaktion bestätigt werden-, sowie zwei nur schwach nachweisbare mit Laufgeschwindigkeiten im Bereich der Mono- und der Diglyceride. Die quantitative Zusammensetzung dieser Mischfraktion wurde durch densitometrische Vermessung der Dünnschichtplatten nach Verkohlung der aufgettennten Substanzkomponenten bestimmt $(0,25 \mathrm{~mm}$ Kieselgel G-Dünnschicht, Ansprühen mit 50proz. wäßr. Schwefelsäure, $45 \mathrm{Min}$. langes Exhitzen auf $180-200^{\circ} \mathrm{im}$ Trockenschrank, Bestimmung der Lichtabsorption im Integraphen der Fa. Zeiss).

Unter Berücksichtigung einer Gesamtsubstanzmenge in dieser Fraktion ron $20 \mathrm{mg}$ ergaben sich die in der Tabelle 4 aufgeführten Werte. Zur gaschromatographischen Untersuchung ihrer Zusammensetzung wurden die freien Fettsäuren auf alkalihaltigen Kieselgelsäulen nach dem Verfahren von MCCARTHY und DUTHIE (14) abgetrennt und dann wie bei den Triglyceriden beschrieben weiterverarbeitet.

Als nächste wurde eine Substanz aus der Säule eluiert, die sich im Laufmittelsystem nach WAGNer (9) wie Cardiolipin oder Phosphatidsäure verhielt. Die Fraktion 5 enthielt Colaminkephalin, die Fraktion 6 Lecithin und die Fraktion 7 Sphingomyelin. Ihre jeweiligen Anteile wurden aus dem Phosphorgehalt ermittelt, nachdem aus dem Gesamtlipoid-P-Gehalt eine Gesamtphosphatidmenge von $86 \mathrm{mg}$ berechnet worden war. Die in den Fraktionen gebundenen Fettsäuren wurden wie oben angegeben gewonnen und auf ihre Zusammensetzung hin untersucht.

Die aus dem Darmgenebe extrahierten Lipide (Tab. 4) bestanden dünnschichtchromatographisch (Abb. 4) fast ausschließlich aus
Tab. 4 Zusammensetzung der aus den Darmlipidfraktionen gewonnenen

\begin{tabular}{lrrrrr}
\hline $\begin{array}{c}\text { Fettsäure*) } \\
(\%)\end{array}$ & $\begin{array}{c}\text { Chol- } \\
\text { esterin- } \\
\text { ester }\end{array}$ & $\begin{array}{c}\text { Trigly- } \\
\text { ceride }\end{array}$ & $\begin{array}{c}\text { Substanz } \\
\text { X }\end{array}$ & $\begin{array}{c}\text { Freie } \\
\text { Fettsäuren }\end{array}$ & $\begin{array}{c}\text { Di- und } \\
\text { Mono- } \\
\text { glyceride }\end{array}$ \\
\hline $12: 0$ & & 1,5 & 2,0 & 1,5 & 5,5 \\
$14: 0$ & 8,0 & 7,0 & 15,5 & 10,5 & 10,5 \\
$16: 0$ & 12,0 & 20,5 & 16,5 & 23,5 & 20,0 \\
$16: 1$ & 5,0 & 6,0 & 6,5 & 7,5 & 4,5 \\
$18: 0$ & 2,5 & 5,0 & 2,0 & 9,5 & 3,5 \\
$18: 1$ & 32,5 & 41,5 & 24,5 & 29,5 & 31,5 \\
$18: 2$ & 10,5 & 13,5 & 14,0 & 11,5 & 14,0 \\
$20: 0-5$ & 4,0 & 3,0 & 5,5 & 3,0 & 3,5 \\
$22: 0-6$ & 2,5 & 1,5 & 2,5 & 1,5 & 2,0 \\
$24: 0-?$ & & & & 1,0 & 3,5 \\
$? * *$ ) & 23,0 & & & & \\
\hline
\end{tabular}

*) Die Zahl vor dem Doppelpunkt gibt die Kettenlänge, die Zahl nach dem Doppelpunkt die Zahl der vorhandenen Doppelbindungen an. **) Rententionsvolumen entspricht etwa 23:0.

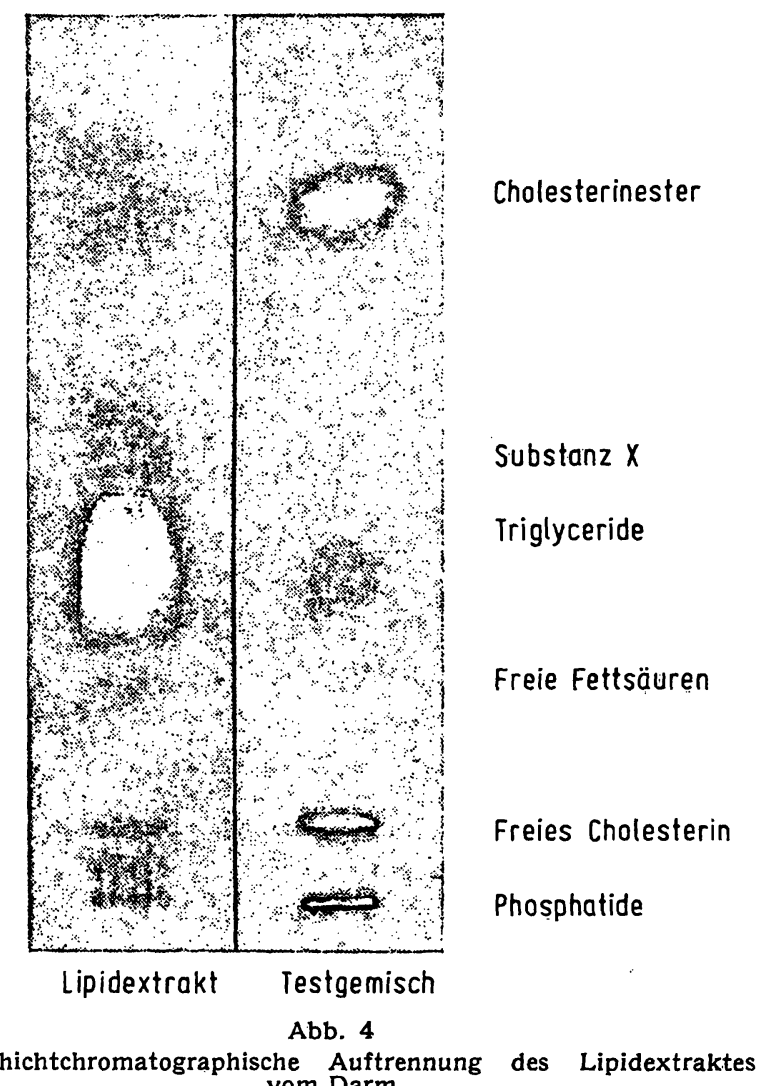
vom Darm

Neutralfett. Zur Anreicherung der Phosphatide wurde deshalb das Material in der Apparatur nach Eberhagen und Betzing (15) gegen Petroläther dialysiert. Aufgrund der dabei erzielten Ausbeuten ergab sich ein Phosphatidgehalt von 1,7\% der Gesamtlipide. Die Phosphatide wurden ebenso wie aliquote Mengen der Neutralfette säulenchromatographisch (siehe oben) aufgetrennt. Aus dem P-Gehalt der Einzelfraktionen errechnete man die Phosphatidzusammensetzung, während die Neutralfettfraktionen zu diesem Zweck umgeestert und dann ihre Acylesterbindungen bestimmt wurden. Die weitere Aufarbeitung erfolgte analog zu den Leberlipiden. In der Triglyceridfraktion fand sich dünnschichtchromatographisch eine schwache Bande mit etwas rascherer Wanderungsgeschwindigkeit als die der Triglyceride, sie wurde in der Abbildung 4 mit X bezeichnet. Die chemische Konstitution dieser Fraktion ist bisher nicht geklärt; nach dem chromatographischen Verhalten könnte es sich um ein Alk(en)yldiglycerid oder um ein Fettsäuren mit ungewöhnlich hoher C-Zahl enthaltendes Triglycerid handeln. 


\section{Besprechung}

Als wichtigstes histologisches Merkmal gilt für den M. WhIPPLE massiver Schaumzellbefall und nur geringgradige chronische Entzündung des Dünndarmstromas. Unsere Beobachtung zeigt das Gegenteil: geringer Schaumzellbefall, hingegen massive chronische Entzündung. Zur Erklärung dieses gegensätzlichen Verhaltens bieten sich an: a priori schütterer Schaumzellbefall, dies stünde in Einklang mit den von ENZINGER und HELwIG (16) angeführten starken graduellen Schwankungen der Dünndarmveränderungen. Ferner könnte die lange Krankheitsdauer, wenigstens 10, vielleicht sogar 20 Jahre, gegenüber einer Durchschnittsdauer des M. WhIPple von 5 Jahren, Makrophagenschwund bedingen, indem der Organismus bei großem Fettmangel auf die in den Makrophagen gehorteten Fettstoffe zurückgreift. Superponierte enteritische Prozesse könnten Makrophagenabbau bedingen. Unseres Erachtens noch überzeugender als im Darm läßt sich der geringe Schaumzellbefall der Lymphknoten auf den chronischen mesenterialen lymphonodulären Rarefizierungsprozeß zurückführen.

Obwohl die im Vergleich zum Darm viel stärker ausgeprägten Veränderungen des lymphatischen Apparates zu dem voreiligen Schluß verleiten, daß ursächlich eine primäre Erkrankung desselben vorliegt, dürfte es sich eher um sekundäre Folgen handeln, die allerdings im Spätstadium das Krankheitsbild beherrschen. Die Lymphknotenveränderungen führen ihrerseits $\mathrm{zu}$ einer progressiven Verstärkung des Malabsorptionssyndromes mit Atrophie und Dystrophie der Dünn- und Dickdarmmukosa, wodurch leichter und häufiger sekundäre enteritische Prozesse entstehen.

Den Tabellen 7 und 8 sind vergleichsweise die eigenen Ergebnisse der Lipiduntersuchungen und jene der Literatur zu entnehmen. Gewisse Unterschiede des Lipidgehaltes und der -zusammensetzung scheinen für den M. Whrpple typisch zu sein. Allerdings bestehen unter den Whipplefällen auch große, zum Teil divergierende Abweichungen. Diese Schwankungen hängen in Anlehnung an Wolman (17) u. a. von der Diät, vom jeweiligen Stoffwechselzustand des Organismus, von der Dauer, der Ausdehnung und Schwere des Befalls der Darmwand und der Lymphknoten sowie vom Vorliegen sekundär enteritischer Prozesse ab. Ferner ist $z u$ berücksichtigen, daß die angewandten Bestimmungsmethoden der einzelnen Autoren stark variieren und daher nur schwer oder gar nicht vergleichbar sind. Da die Details aus den Tabellen hervorgehen, werden lediglich die für den M. WhIPple charakteristischen Merkmale betont.

\section{Darmlipide (Tab. 5):}

Wenn auch Hendrix und Mitarbeiter (18) eigenartigerweise bei ihren Fällen eher erniedrigte Werte finden, ist sicherlich eine - bei uns excessive - Vermehrung der Gesamtlipide ein Charakteristikum des M. WhIPpLE. Innerhalb der Gesamtlipide sind die Triglyceride massiv vermehrt. Diese ausgeprägte Neutralfettanhäufung im eigenen Fall erklärt wohl auch viele Unterschiede zu

Tab. 5

Darmlipide (Angaben in \%)

Übersicht über die eigenen Ergebnisse und Vergleichsangaben aus der Literatur

\begin{tabular}{|c|c|c|c|c|c|c|c|c|c|}
\hline \multirow{3}{*}{$\begin{array}{l}\text { Lipidfraktion } \\
\text { Gesamtlipide } \\
\text { Cholesterin-Ester }\end{array}$} & \multirow{3}{*}{$\begin{array}{c}\begin{array}{c}\text { bezogen } \\
\text { auf }\end{array} \\
\text { FG } \\
\text { FG } \\
\text { GL } \\
\text { GL-TG } \\
\text { FG-TG }\end{array}$} & \multirow{3}{*}{$\begin{array}{c}\begin{array}{c}\text { Eigene } \\
\text { Werte }\end{array} \\
26,7 \\
0,24 \\
0,91 \\
8,7 \\
0,3\end{array}$} & \multirow{2}{*}{$\begin{array}{c}\begin{array}{c}\text { ROSEN und } \\
\text { ROSEN } \\
(43)\end{array} \\
53,4\end{array}$} & \multirow[t]{2}{*}{$\begin{array}{c}\text { FitZGERALD } \\
\text { und KINNEY } \\
(44)\end{array}$} & \multicolumn{4}{|c|}{$\begin{array}{l}\text { HENDRIX und Mitarbeiter (18) } \\
\text { Fall } 1\end{array}$} & \multirow{3}{*}{$\begin{array}{c}\begin{array}{c}\text { Normalwerte } \\
\text { NormDERS und } \\
\text { Mitarbeiter (45) } \\
\text { Mitand }\end{array} \\
4,2 \pm 0,9\end{array}$} \\
\hline & & & & & 2,26 & 1,6 & 2,22 & 1,79 & \\
\hline & & & & & $\begin{array}{l}0,07 \\
3,0 \\
6,4 \\
0,05\end{array}$ & $\begin{array}{l}0,08 \\
5,0 \\
7,0 \\
0,09\end{array}$ & $\begin{array}{c}0,11 \\
5,0 \\
13,0 \\
0,11\end{array}$ & $\begin{array}{l}0,7 \\
3,9 \\
8,3 \\
0,04\end{array}$ & \\
\hline Freies Cholesterin & $\begin{array}{l}\text { FG } \\
\text { GL } \\
\text { GL-TG } \\
\text { FG-TG }\end{array}$ & $\begin{array}{l}0,16 \\
0,61 \\
5,4 \\
0,2\end{array}$ & · & & $\begin{array}{c}0,25 \\
11,4 \\
22,1 \\
0,25\end{array}$ & $\begin{array}{r}0,2 \\
12,9 \\
18,9 \\
0,2\end{array}$ & $\begin{array}{c}0,18 \\
7,9 \\
20,6 \\
0,18\end{array}$ & $\begin{array}{c}0,16 \\
9,4 \\
20,0 \\
0,16\end{array}$ & \\
\hline $\begin{array}{l}\text { Gesamt-Chol- } \\
\text { esterin }\end{array}$ & $\begin{array}{l}\text { FG } \\
\text { GL } \\
\text { GL-TG } \\
\text { FG-TG }\end{array}$ & $\begin{array}{c}0,4 \\
1,52 \\
13,5 \\
0,5\end{array}$ & 7,8 & 0,48 & $\begin{array}{c}0,32 \\
14,2 \\
28,4 \\
0,3\end{array}$ & $\begin{array}{c}0,28 \\
18,0 \\
26,4 \\
0,3\end{array}$ & $\begin{array}{c}0,29 \\
13,1 \\
33,6 \\
0,3\end{array}$ & $\begin{array}{c}0,23 \\
13,3 \\
28,3 \\
0,2\end{array}$ & $\begin{array}{l}7,7 \pm 1,9 \\
10,1 \pm \\
0,42\end{array}$ \\
\hline Triglyceride & $\begin{array}{l}\mathbf{F G} \\
\mathbf{G L}\end{array}$ & $\begin{array}{l}23,7 \\
88,8\end{array}$ & & & $\begin{array}{l}1,13 \\
50,0\end{array}$ & $\begin{array}{r}0,5 \\
31,8\end{array}$ & $\begin{array}{l}1,35 \\
61,1\end{array}$ & $\begin{array}{r}0,9 \\
53,0\end{array}$ & $22,8 \pm 8,5$ \\
\hline Freie Fettsäuren & $\begin{array}{l}\text { FG } \\
\text { GL } \\
\text { GL-TG } \\
\text { FG-TG }\end{array}$ & $\begin{array}{r}1,1 \\
4,3 \\
41,0 \\
1,5\end{array}$ & 10,6 & & $\begin{array}{c}0,13 \\
6,0 \\
11,6 \\
0,13\end{array}$ & $\begin{array}{l}0,06 \\
3,8 \\
5,7 \\
0,06\end{array}$ & $\begin{array}{l}0,05 \\
2,0 \\
5,4 \\
0,05\end{array}$ & $\begin{array}{l}0,07 \\
4,3 \\
9,2 \\
0,08\end{array}$ & $\begin{array}{l}0,134 \\
3,2 \pm 2,9 \\
4,2 \\
0,2\end{array}$ \\
\hline Phosphatide. & $\begin{array}{l}\text { FG } \\
\text { GL } \\
\text { GL-TG } \\
\text { FG-TG }\end{array}$ & $\begin{array}{c}0,44 \\
1,67 \\
15,8 \\
0,6\end{array}$ & 9,1 & 2,28 & $\begin{array}{c}0,12 \\
5,5 \\
11,0 \\
0,12\end{array}$ & $\begin{array}{l}0,09 \\
5,7 \\
8,2 \\
0,09\end{array}$ & $\begin{array}{l}0,08 \\
4,3 \\
9,2 \\
0,08\end{array}$ & $\begin{array}{l}0,07 \\
4,3 \\
9,2 \\
0,08\end{array}$ & $\begin{array}{r}2,5 \\
49,6 \\
65,0 \\
2,7\end{array}$ \\
\hline $\begin{array}{l}\text { Colamin- } \\
\text { Kephalin }\end{array}$ & $\begin{array}{l}\text { FG } \\
\text { GL } \\
\% \text { PL } \\
\text { GL-TG }\end{array}$ & $\begin{array}{c}0,43 \\
25,7 \\
3,8\end{array}$ & & & & & & & $\begin{array}{l}0,42 \\
10,1 \\
20,4 \pm 3,1\end{array}$ \\
\hline Lecithin & $\begin{array}{l}\mathrm{FG} \\
\mathrm{GL} \\
\% \mathrm{PL} \\
\text { GL-TG }\end{array}$ & $\begin{array}{l}1,08 \\
64,7 \\
10 ; 3\end{array}$ & & & & & & & $\begin{array}{l}0,89 \\
21,3 \\
43,0 \pm 3,4\end{array}$ \\
\hline Sphingomyelin & $\begin{array}{l}\text { FG } \\
\text { GL PL }\end{array}$ & $\begin{array}{l}0,16 \\
9,6\end{array}$ & & & & & & & $\begin{array}{l}+ \text { Inosithol } \\
0,35 \\
8,33 \\
16,8 \pm 1,2\end{array}$ \\
\hline
\end{tabular}

Zeichenerklärung: $F G=$ Feuchtgewicht, $G L=$ Gesamtlipide, $T r G=$ Trockengewicht, $T G=$ Triglyceride, $P L=$ Plısphatide,

GL-TG = bezogen auf Gesamtlipide $\}$ abzüglich der Triglyceride 
Tab. 6

Leberlipide (Angaben in \%)

Vergleich der eigenen Ergebnisse mit Angaben aus der Literatur

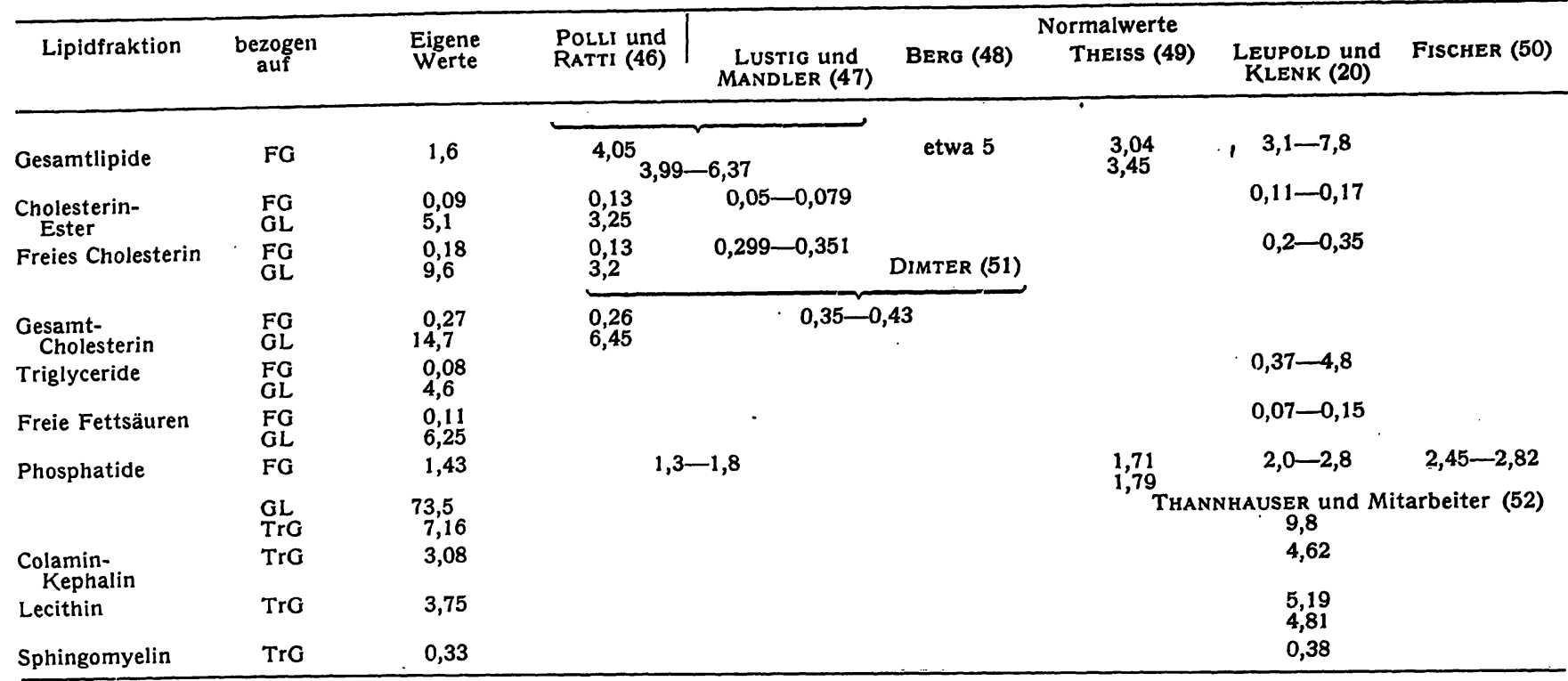

Zeichenerklärung: $F G:=$ Feuchtgewicht, $G L=$ Gesamtlipide, $\operatorname{Tr} G=$ Trockengewicht

den Befunden anderer Autoren. Es ist daher für die Cholesterinverte keine sichere Aussage möglich, zum Teil dürften die Unterschiede auch innerhalb der Fehlerbreite liegen. Die Erhöhung der freien Fettsäuren läßt sich entweder durch einen verringerten Einbau der Fettsäuren in Triglyceride oder durch postmortale Lipolyse erklären. Durch die bereits 7 Stdn. nach dem Tod des Patienten exfolgte Obduktion und die sofortige Aufbewahrung des Gewebsmaterials bis zur Aufarbeitung bei $-20^{\circ}$ dürften autolytische Vorgänge allerdings quantitativ weitgehend eine untergeordnete Rolle spielen. Die Phosplatide zeigen eine deutliche Verminderung, wobei alle Phosphatidfraktionen gleichmäßig betroffen sind. Es ist anzunehmen, daß die angehäuften Triglyceride die anderen Zellbestandteile wie Gewebswasser (wichtig für den Bezug auf das Feuchtgewicht), die phosphatidreichen Mitochondrien, Mikrosomen und das Protoplasma zurückdrängen. In Anlehnung an die Erfahrungen von MASSHOFF und Mitarbeitern (19) erhebt sich bei den Phosphatiden die Frage nach deren Verminderung durch postmortale Autolyse, desgleichen bei der Vermehrung der freien Fettsäuren. Gegen die Annahme postmortaler Einflüsse spricht der Befund von HendRIX und Mitarbeitern (18) die auch erniedrigte Phosphatide, aber keine erhöhten freien Fettsäuren finden. Darüber hinaus ist in Anbetracht des Ausbleibens einer Vermehrung der Lysophosphatide der Einfluß der Autolyse wohl zu vernachlässigen. Mit Sicherheit ließe sich diese Frage nur durch entsprechende Vergleichsuntersuchungen an normalen Darmgewebe mit gleichzeitiger Bestimmung des freien Glycerins beantworten. Die gaschromatographische Analyse der Fettsäuren innerhalb der Triglyceride zeigt einerseits eine deutliche Verminderung der Palmitin-, Stearin- und der Polyenfettsäuren, andererseits eine Erhöhung der Myristinund Palmitoleinsäuren (Tab. 7a).
Leberlipide (Tab. 6):

Auch bei diesen halten wir einen wesentlichen Einfluß der Autolyse auf die Lipidwerte für wenig wahrschein-

Tab. 7

Fettsäurezusammensetzung (Angaben in \%) a) Darm: Triglyceride

\begin{tabular}{ccc}
\hline Fettsäuren & $\begin{array}{c}\text { Eigene } \\
\text { Ergebnisse }\end{array}$ & $\begin{array}{c}\text { Normalwerte } \\
\text { SAUNDERS und } \\
\text { Mitarbeiter (45) }\end{array}$ \\
\hline $12: 0$ & 1,5 & \\
$14: 0$ & 7,0 & \\
$16: 0$ & 20,5 & $31,8 \pm 4,4$ \\
$18: 0$ & 5,0 & $11,9 \pm 1,2$ \\
$16: 1$ & 6,0 & $3,7 \pm 1,1$ \\
$18: 1$ & 41,5 & $35,3 \pm 4,8$ \\
$18: 2$ & 13,5 & $13,7 \pm 6,2$ \\
\hline
\end{tabular}

b) Leber: Triglyceride

\begin{tabular}{|c|c|c|c|c|}
\hline Fettsäure & $\begin{array}{l}\text { Eigene } \\
\text { Werte }\end{array}$ & $\begin{array}{l}\text { LEUPOLD } \\
\text { und } \\
\text { KLENK (20) }\end{array}$ & $\begin{array}{c}\text { Normalwerte } \\
\text { TAKAHASHI } \\
\text { und } \\
\text { TANAKA (53) }\end{array}$ & BERG (48) \\
\hline $\begin{array}{l}12: 0 \\
14: 0 \\
16: 0 \\
16: 1 \\
18: 0 \\
18: 1 \\
18: 2 \\
20: 0 \\
20: 3 \\
20: 4 \\
22: 0-6 \\
20 \text { ungesättigt }\end{array}$ & $\begin{array}{r}1,5 \\
9,5 \\
20,0 \\
5,0 \\
6,0 \\
36,5 \\
10,5 \\
0,5 \\
1,0 \\
2,5 \\
3,5\end{array}$ & $\begin{array}{c}1,1 \\
23,24 \\
9,5 \\
3,4 \\
56,52 \\
6,12 \\
\\
2,2\end{array}$ & $\begin{array}{r}1,4 \\
29,2 \\
6,4 \\
9,1 \\
35,9 \\
15,9\end{array}$ & $\begin{array}{r}26,9 \\
6,7 \\
10,1 \\
24,9 \\
8,4 \\
\\
0,5\end{array}$ \\
\hline
\end{tabular}

c) Leber: Freie Fettsäuren

\begin{tabular}{ccc}
\hline Fettsäuren & Eigene Werte & $\begin{array}{c}\text { Normalwerte } \\
\text { LEUPOLD und } \\
\text { KLENK (20) }\end{array}$ \\
\hline $12: 0$ & 1,0 & \\
$14: 0$ & 26,0 & 1,2 \\
$16: 0$ & 19,0 & 22,27 \\
$16: 1$ & 2,5 & 8,4 \\
$18: 0$ & 10,5 & 9,16 \\
$18: 1$ & 19,5 & 51,22 \\
$18: 1$ & 10,0 & 7,26 \\
$20: 0$ & 1,5 & 1,2 \\
$20: 3$ & 3,0 & Spur \\
$20: 4$ & 4,5 & 1,1 \\
$22: 0-6$ & &
\end{tabular}

Z. klin. Chem. u. klin. Biochem. / 8. Jahrg. 1970/ Heft 5 
Tab. 8

1. Lymphknotenlipide

a) Flüssiger Cyteninhalt (eigene Werte): Triglyceride $1030 \mathrm{mg} / 100 \mathrm{ml}$, Cholesterin $140 \mathrm{mg} / 100 \mathrm{ml}$, Phosphatide $188 \mathrm{mg}$ ! $100 \mathrm{ml}$ b) Solider Lymphknoteninhalt: Vergleichswerte aus der Literatur (Lipiduntersuchungen an Lymphknoten bei M. Whipple) Angaben in $\bullet$

\begin{tabular}{|c|c|c|c|c|c|c|c|c|c|c|}
\hline \multirow{2}{*}{$\frac{\text { Lipidfraktion }}{\text { Gesamtlipide }}$} & \multirow{2}{*}{$\begin{array}{c}\begin{array}{c}\text { bezogen } \\
\text { auf }\end{array} \\
\text { FG } \\
\text { TrG }\end{array}$} & \multirow[t]{2}{*}{$\begin{array}{l}\text { Eigene } \\
\text { Werte }\end{array}$} & \multirow[t]{2}{*}{$\underset{\text { (2) }}{\text { WhIPPLE }}$} & \multicolumn{2}{|c|}{$\begin{array}{l}\text { HENDRIX und } \\
\text { Mitarbeiter (18) }\end{array}$} & \multirow{2}{*}{ ROSEN (43) } & \multirow{2}{*}{$\begin{array}{c}\text { PLuMRER } \\
(21) \\
31,2\end{array}$} & \multicolumn{3}{|c|}{$\begin{array}{l}\text { CASSELbLAN }(54) \\
\text { M. Whipple Normalwerte }\end{array}$} \\
\hline & & & & 31 & 22 & & & 14,9 & 16,4 & $28,1-53,8$ \\
\hline Triglyceride & $\underset{\mathbf{G G}}{\mathbf{F L}}$ & 3,2 Gew. :; & 22 & $\begin{array}{l}24 \\
78\end{array}$ & 77 & & 21,1 & & & \\
\hline Cholesterin & $\begin{array}{l}\text { FG } \\
\text { GL } \\
\text { TrG }\end{array}$ & 5,8 Gew. \% & $D$ & $11^{3,5}$ & 29 & $\begin{array}{l}8,2 \\
6,1\end{array}$ & & 7,9 & 9,3 & $0,4-1,2$ \\
\hline \multirow[t]{2}{*}{ Phosphatide } & FG & 8,0 Gew. $:$ : & $\begin{array}{l}\text { kein } \\
\text { Lecithin }\end{array}$ & $\mathbf{0}$ & $\mathbf{0}$ & $<2$ & & & & \\
\hline & GL & & & & & $\begin{array}{l}7.9 \\
5,8\end{array}$ & & 1,98 & 3,95 & $0,9-2,9$ \\
\hline Gesamt Fettsāuren & $\begin{array}{l}\text { FG } \\
\text { GL } \\
\text { TrG }\end{array}$ & & & 68 & 64 & $\begin{array}{l}87 \\
64\end{array}$ & 24,4 & & & \\
\hline Freie Fettsäuren & $\begin{array}{l}\text { FG } \\
\text { GL } \\
\text { TrG }\end{array}$ & & & 6,7 & 10,4 & $\begin{array}{l}16,4 \\
12,1\end{array}$ & 1,3 & & & \\
\hline $\begin{array}{l}\text { Gesamtlipide/trockenem } \\
\text { fettreiem Rückstand }\end{array}$ & & & & & & & & 1,96 & $1, \overline{52}$ & $4,7-8,3$ \\
\hline $\begin{array}{l}\text { Glyceride und freie } \\
\text { Fettsāuren } \\
\text { Verseifungszahl }\end{array}$ & GL & & 144,4 & & & 166 & & 86 & 84 & $95-99$ \\
\hline
\end{tabular}

Zeichenerklārung: FG = Feuchtgewicht, $\mathbf{G L}=$ Gesamtlipide, TrG = Trockengewicht

2. Chylöser Ascites

\begin{tabular}{lcc}
\hline Lipidfraktion & Eigene Werte & PETERsox und KAMPMEIER (55) \\
\hline Triglyceride & $72 \mathrm{mg} / 100 \mathrm{ml}$ & \\
$\begin{array}{l}\text { Cholesterin } \\
\text { Phosphatide }\end{array}$ & $45 \mathrm{mg} / 100 \mathrm{ml}$ & $33 \mathrm{mg} / 100 \mathrm{ml}$ \\
Fettgehalt & $89 \mathrm{mg} / 100 \mathrm{ml}$ & $1630 \mathrm{mg} / 100 \mathrm{ml}$ \\
EiweiB & & $66 \mathrm{mg} / 100 \mathrm{ml}$ \\
\hline
\end{tabular}

lich, wenn man die Ergebnisse von LEuPOLD und KIENK (20) berücksichtigt. Für die Leberlipide fehlen uns Vergleichsangaben von Whipplekranken. Der Gesamtlipidgebalt und die Triglyceridfraktion sind in unserem Fall deutlich vermindert, das Gesamtcbolesterin scheint eine leichte Emiedrigung aufzuweisen. Die Pbospbatidwerte liegen niedrig, die einzelnen Fraktionen scheinen gleichmāßig betroffen zu sein. Innerhalb der Triglyceridfraktion ist der Anțeil der Myristinsāure erhöht, wāhrend die Palmitinsāure leicht emiedrigt ist, die Stearinsāure im Normbereich und die Palmitoleinsāure im unteren Normbereich liegen. Der Wert der Ölsāure und Linolsāure entsprechen der Norm. Die Fraktion der freien Fettsāuren weist einen deutlich erhöhten Mỵristingehalt auf.

\section{Lympblenotenlipide (Tab. 8, 1):}

Obwohl einige Autoren Ljmphknoten von Whipplekranken lipidchemisch untersucht haben; sind die Werte schlecht vergleichbar. Der Cholesteringehalt ist sicher, der Phosphatidwert möglicherweise erhöht. Die niedrige Verseifungszahl spricht für das Vorhandensein von langkettigen Fettsäuren.

\section{Cbylöser Aszites (Tab. 8, 2):}

Pruasar und Mitarbeiter (21) geben in 5 Fällen ron M. Whipple dreimal das Vorhandensein eines solchen an. Die in der Literatur gefundenen Werte reichen zum Vergleich nicht aus.

\section{Serumlipide:}

Bei unserem Fall sind die Werte des Serumcholesterin, der Triglyceride und der Phosphatide deutlich erniedrigt. Auf Grund ihrer eigenen Ergebnisse und ron Literaturangaben geben ENzLiger und HeLwig (16) die Serumcholesterinwerte bei 34 von 43 Patienten, bei 3 Parienten sogar betrāchulich erniedrigt an. LASTER und Mitarbeiter (22) sehen ebenfalls niedrige Cholesterinuerte. Wie ENzLiger und Mitarbeiter (16) anführen, betragen die Gesamtserumlipoide bei 5 von 11 Patienten weniger als $500 \mathrm{mg} / 100 \mathrm{~m} /$. Auch diese Autoren machen keine Angaben über Serumtriglyceridbestimmungen, so da $B$ anzunehmen ist, daß bisher bei Whipplekranken diese Fraktion noch kaum mit einer verläßlichen Methode bestimmt wurde. Bei einem Teil der Fālle finden Plunarer und Mitarbeiter $(21,23)$ erniedrigte Werte der Gesamtserumlipoide und des Serumcholesterins. WEIGEL und SFIES (24) berichten bei einem Fall über das Ausbleiben des üblichen Anstieges der Serumfette nach einer Mrahlzeit.

\section{Substanz $X$ :}

Ein überaus interessanter Befund der rorliegenden Untersuchung ist der Nachweis einer abnormen Lipoidfraktion in der Darmwand unseres Whipplekranken. Schon WhIPPLE (2) diskutierte die pathogenetische Rolle „eines entweder in sich abnormen Fettes oder eines Fettes, das eine abnorme oder toxische Substanz enthält". Wenn auch die genaue Identitāt der Substanz X 
noch nicht feststeht und ihre Klärung weiteren Analysen vorbehalten ist, muß betont werden, $\mathrm{da} ß$ es sich mit größter Sicherheit um kein Artefakt haridelt. Auch soll vor Abschluß der Untersuchungen keine Stellung zur Bedeutung dieser doch nur $1,2 \%$ der Gesamtlipide in der Darmwand ausmachenden Fraktion genommen werden.

In diesen Ausführungen bleibt kein Raum, näher auf die verschiedenen Theorien über die Ursache und die Pathogenese des M. Whipple einzugehen. Auch wir sind mit den von uns erhobenen Befunden nicht imstande, eine dezidierte Aussage über die Ätiologie zu machen. Wohl gewähren unsere Ergebnisse einen gewissen Einblick in die Stoffwechselvorgänge beim M. Whipple. Zusammen mit den Angaben aus der Literatur helfen unsere Resultate vielleicht, die pathogenetischen Zusammenhänge besser zu verstehen.

Seit den Untersuchungen von SIERACKI (25) wird von vielen Autoren aufgrund lichtmikroskopischer (SIEracki und Fine (26), Meesen (27). Schmitt und Mitarbeiter (28)), elektronenmikroskopischer (CAROLI und Mitarbeiter (29), KENT und Mitarbeiter (30), KURTZ und Mitarbeitè (31), Müller und Kemmer (32), CoheN und Payne (33), Dobbins und Ruffin (34)) und tierexperimenteller Beobachtungen (MüLLER und SCHLOTTERHOSZ (35)) ein infektiöses Agens, in der Regel Bakterien, als Ursache des M. Whipple angesehen, da man solche in den Darmepithelien, in der Lamina propria des Dünndarms, in den Lymphgefäßepithelien, in mesenterialen und anderen Lymphknoten, sogar in anderen Organen, also ubiquitär, fand.

Wir konnten lichtmikroskopisch keine Bakterien nachweisen, ebenso fanden sich in den PAS-positiven Zytoplasmaeinschlüssen der Makrophagen keine eindeutigen "Sieracki-Partikel“ (die als phagozytierte Bakterienabbauprodukte gelten). Der negative Bakteriennachweis könnte darauf beruhen, daß die Paraffinschnitte hierfür ungeeignet sind (siehe TRIER und Mitarbeiter (36)); wir sind aber eher der Ansicht, daß Antibiotika- und Cortisontherapie Bakterienschwund bedingten (DRUBE und WIDGREN (37)).

Die infektiös-bakterielle Theorie des M. Whipple weist mehrere schwache Stellen auf. Es liegt weder ein einheitlicher Erreger vor - CAROLI und Mitarbeiter (29): anaerobes Corynebakterium, KJHAERHEIM und Mitarbeiter (38) Hämophilusstamm - noch gelang es mit den genannten Keimen, einen $\mathrm{M}$. Whipple experimentell $z u$ erzeugen. Ferner ist unklar, ob die angeschuldigten Keime den Darm primär oder erst sekundär befallen, und was für eine Schädigung sie am Darm hervorrufen. Denkbar wäre, daß durch den Keimbefall des Dünndarmepithels eine mechanische, durch den Abbau und allenfalls durch die toxische Wirkung der Keime auch eine funktionelle (Teil-)Blockade der Zelle erfolgt, wobei bisher unbekannte endozelluläre (erworbene?) enzymatische Störungen eine Rolle spielen könnten. Unsere Úberlegungen stünden in Einklang mit der Tatsache, daß durch Antibiotika- und Cortisontherapie nicht nur bioptisch gesicherter Bakterienschwund, sondern auch
Remission des Leidens erfolgt. Widersprüchlich sind auch die Ansichten über den Angriffspunkt der Fettstoffwechselstörungen. ENZINGER und HeLwig (16) folgern aufgrund von Untersuchungen an Whipplepatienten, bei denen die Resorption von ${ }^{131} \mathrm{~J}$ markierten Triolein und von màrkierter Ölsäure beträchtlich gestört waren, daß die Resorption für Lipide beeinträchtigt sei. Der elektronenmikroskopische Nachweis unveränderter Mikrovilli der Dünndarmepithelien (CoHEN (39)) spricht u. E. (s. oben) nicht gegen eine Resorptionsstörung. Ferner kommt hinzu, daß bei Spätformen des M. Whipple wie in unserem Falle infolge superponierter chronischer Entzündungsprozesse oder/und funktioneller Erschöpfung der Darmepithelien mit zusätzlicher Epithelschädigung und Resorptionsstörung gerechnet werden muß. BRICE und Mitarbeiter (40) finden bei Whipple-Kranken einen gestörten Aminosäurestoffwechsel und eine verringerte Fähigkeit des Dünndarmepithels, langkettige Fettsäuren zu verestern, betonen aber bei der Diskussion ihrer in-vitro-Untersuchungen, daß der Nachweis eines funktionellen Defektes des Darmepithels nicht unbedingt von erstrangiger Bedeutung für die Pathogenese der Malabsorption sein müsse. Besonders der Befund eines erhöhten Triglyceridgehaltes der Darmmukosa - wie ihn unsere Ergebnisse deutlich zeigen - würde für das gleichzeitige Vorliegen eines behinderten Lymphtransportes von veresterten langkettigen Fettsäuren sprechen. DobBins und Ruffin (34) lassen die Frage offen, ob sowohl die verminderte Aminosäureaufnahme und Fettsäureveresterung des Dünndarmepithels in vitro als auch die Blockierung der Chylomikronenabsorption nicht Folgeerscheinungen der Makrophagenansammlung in der Lamina propria darstellen. Da die Theorie der Resorptionsstörung als Ursache des M. Whipple nicht ganz befriedigt, wird in jüngster Zeit (TRIER (41), Henning und Berg (42)) eine Störung des Transportes der Chylomikronen angenommen, wobei das liegengebliebene Fett von den Makrophagen des Dünndarmstromas, $z$. T. auch erst in den mesenchymalen Lymphknoten, resorbiert wird. Zur Klärung dieses Fragenkomplexes scheinen frische Formen des M. Whipple besser geeignet als Spätformen, wie unsere Beobachtung, da hier ursprüngliches Geschehen und Folgeerscheinungen zu sehr miteinander verquickt sind. Die ins Auge springende Akkumulation der Gesamtlipide in der Darmwand ist hauptsächlich durch die Triglyceridvermehrung verursacht. Dadurch wird auch vermutlich die Zurückdrängung der Zellipide (Phosphatide) her-. vorgerufen. Die qualitativen Verschiebungen der Fettsäuren innerhalb der Triglyceride sind schwierig zu interpretieren. Sehr wahrscheinlich liegt aber kein absoluter Mangel an den relativ verringerten Fettsäuren in dieser Fraktion vor. Die qualitative Abweichung gegenüber Normalwerten ist dennoch beachtenswert. Für diese Anhäufung von Lipiden sind sowohl die pathologischen Veränderungen des intestinalen und mesenchymalen Lymphsystems verantwortlich. Mit der Malabsorption stehen sicherlich auch die Leberveränderungen in Zusammenhang. Die. Leberbeteiligung äußert 
sich einerseits in den alterierten Lipidwerten dieses Organs, andererseits können die erniedrigten Serumlipidfraktionen wohl als Ausdruck einer verminderten Synthese in der Leber gedeutet werden. Eindrucksvol haben LASTER und Mitarbeiter (22) bei Whipplekranken die Normalisierung des vorher - während der Exarcerbation der Erkrankung - erniedrigten Serumcholesterinspiegels in der Remission gezeigt. In den verminderten Lebertriglyceriden fällt eine qualitative Abweichung des Fettsäuremuster auf, wobei - wie im Darm - die
Palmitinsäure verringert und die Myristinsäure erhöht sind. In der Fraktion der freien Fettsäuren fällt die starke Vermehrung der Myristinsäure auf.

Obwohl unsere Untersuchungsergebnisse zugunsten einer primären Resorptionsstörung sprechen, liegt eine kombinierte Störung der Resorption und des Transportes der Lipoide pathogenetisch beim $M$. Whipple durchaus im Bereiche der Möglichkeit, wobei allerdings die Transportstörung nachgeschaltet sein dürfte.

\section{Literatur}

1. Scrmid, K. O., Verh. Dtsch. Ges. Path. 53. Tagg., Mainz, 163-168 (1969). - 2. Whupple, G. H., John Hopkins Hosp. Bull. 198, 382 (1907). - 3. FolCh, J., M. LeES und G. H. SLOANEStANLEY, J. biol. Chemistry 226, 497 (1957). - 4. BartLETt, G. R., J. biol. Chemistry 234, 466 (1959). - 5. ZAK, B., N. Moss, A. J. Boyle und A. Zlatkis, Analytic. Chem. 26, 776 (1954). 6. Fried, R. und J. Hoeflyayr, Klin. Wschr. 41, 727 (1963). 7. Eberhagen, D., G. Plette und O. Hauck, Zschr. exper. Med. 145, 171 (1968). - 8. Zollner, N. und G. Wolfram, Klin. Wschr. 40, 1101 (1962). - 9. WAGNER, H., Fette Seifen einschl. Anstrichmittel 62, 1115 (1960). - 10. SKIPSKr, V. P., R. F. Peterson und M. Barclay, Biochem. J. 90, 374 (1964). 11. Eberhagen, D. und U. Remler, Strahlentherapie 132, 441 (1967). - 12. Eberhagen, D. und G. Plette, Hoppe-Seyler's Zschr. physiol. Chem. 343, 17 (1965). - 13. EberhageN, D. Z., analyt. Chem. 212, 230 (1965). - 14. Mc Carthy, R. D. und A. H. Duthie, J. Lipid Res. 3, 117 (1962). - 15. Eberhagen, D. und H. BetzIng, J. Lipid Res. 3, 382 (1962). - 16. ENZINGer, F. M. und E. B. Helwig, Virchow's Arch. pathol. Anatom. Physiol. klin. Med. 336, 238 (1963). - 17. Wolaran, M., in: Handbuch der Histochemie, herausgegeben von Grauman, W. und K. NeunanN, Volume V., Lipides, Second Part, Histochemistry of Lipids in Pathology, Gustav Fischer Verlag, Stuttgart (1964). - 18. Hendrix, S. P., B. BalCK-Schaffer, R. W. Wrutheres und P. Handlek, Arch. Int. Med. Chicago 85, 91 (1950). - 19. Masshoff, W., F. Lindlar und H. J. StolpasanN, Virchow's Arch. pathol. Anatom. Physiol. klin. Med. 337, 340 (1964). - 20. Leupold, F. und H. D. Krenk, in: Dtsch. Arch. klin. Med. 210, 238 (1965). - 21. Plummer, K., B. B. Wersiger III, C. M. Caravati und H. S. Kupperman, Atch. Int. Med. Chicago 91, 784 (1953). - 22. Laster, L., T. A. WaLDMANN, L. F. Fenster und J. W. Singleton, J. Clin. Invest. 45, 637 (1966). 23. Plummer, K., S. Russi, W. H. jr. Harris und C. M. Caravati, Arch. Int. Med. Chicago 86, 280 (1950). - 24. WeIGeL, H. und B. SpIEs, Dtsch. Arch. klin. Med. 203, 66 (1956). - 25. SierACKI, J. C., Arch. Pathol., Chicago 66, 464 (1958). - 26. SierAcki, J. C. und G. Fine, Arch. Pathol. Chicago 67, 81 (1959). - 27. Meesen, H., Dtsch. Med. Wschr. 89, 1760 (1964). - 28. Schmrt, W., H. Becker, D. C. Platt und G. Beneke, Med. Welt 47,
2571 (1966). - 29. Cyroli, J., A. R. Prevot, C. Julien, M. Sebald und J. Eteve, Compt. rend. Acad. sc., Paris 256, 329 (1963). - 30. Kent, T. H., J. M. Layton, J. A. Cliffon und H. P. Schedel, Laborat. Invest. 12, 1163 (1963). - 31. KuRTZ, S. M., T. D. jr. Davis und J. M. Rufrin, Laborat. Invest. 11, 653 (1962). - 32. Müller, M. und C. Kearmer, Zbl. allg. Path. 107, 488 (1965). - 33. Cohen, L. M. und T. P. B. Paine, N. Y. State J. Med. 66, 2148 (1966). - 34. Dobrins III, O. W. und J. R. Rufrin, Amer. J. Pathol. 51, 225 (1967). - 35. MüLLER, MI. und L. Schlotter hosz, Zbl. allg. Path. 109, 46 (1966). - 36. Thier, S. J., P. C. Phelps, S. Eidelaran und C. E. Rubin, Gastroenterology, Baltimore 48, 684 (1965). - 37. Drube, H. C. und S. WILDGREN, Schweiz. med. Wschr. 97, 9 (1967). - 38. KJAERHEIM, A., T. Midtvedt, S. SKrede und E. G. Jone, Acta path. microbiol. Scand. 66, 135 (1966). - 39. Cohen, S. A., J. Ultrastructure Res. 10, 124 (1964). - 40. Brice, R. S. jr., E. E. Cwen und M. P. TYOR, Gastroenterology, Baltimore 48, 584 (1965). - 41. THIER, S. J., Fed. Proc. 26, 1391 (1967). - 42. Henning, N. und G. BerG, in: Verdauung und Resorption in HeILMEYER's Lehrbuch der speziellen pathologischen Physiologie, 11. Aufl. G. Fischer Verlag, Stuttgart (1968). - 43. Rosen, M. S. und S. H. Rosen. Amer. J. Pathol. 23, 443 (1947). - 44. Frzgerald, P. J. und T. P. KinNey. Amer. J. Pathol. 21, 1069 (1945). - 45. Saunders, D. R., P. O. Ways, C. M. Parmentier und C. M. Rubin. J. Chir. Invest. 45, 1516 (1966). - 46. Polli, E und G. Rattr. Biochem. Z. 321, 166 (1950). - 47. LustrG, B. und E. MandLER. Biochem. Z. 249, 352 (1932). - 48. BERG, G., in Fettleber, Internat. Symposium an der Med. Klinik mit Poliklinik der Univ. Erlangen-Nürnberg in Erlangen, 17. und 18. März 1967. Pallas Verlag, Lochham bei München (1968) p. 22. - 49. TheIss, E. R., J. biol. Chemistry 82, 327 (1929). - 50. FrsCHER, A., Biochem. Z. 175, 449 (1926). 51. Dimter, A. zit. n., H. D. Cremer und Z. Führ, in HoppeSeyler/Thierfelder Bd. 5, p. 447, Springer Verlag Berlin. 52. Thannhauser, S. J., J. Benotti, A. Walcott und R. ReinSTEIN, J. biol. Chemistry 129, 717 (1939). - 53. Takafashi, Y. und T. Tanaka, J. Biochem. (Tokyo) 49, 713 (1961). - 54. Casselman, W. G. B., A. I. Macrae und E. H. Simmons. J. Path. Bact. 68, 67 (1945). - 55. Peterson, J. C. und R. H. Kampmieier. Amer. J. Med. Sc. 221, 543 (1951).

Doz. Dr. K. O. Schmid

A 8036, Graz, Landeskrankenhaus, Pathol. anat. Institut Dr. P. Spath, Innsbruck, Medizinische Universitätsklinik

Priv. Doz. Dr. D. Eberhagen

8 München 12, Westendstr. 267

Prim. Dr. M. Ciresa

I-andeskrankenhaus Schwaz/Tirol, Med. Abteilung 